\title{
Acquiring field data and creating vibration tests
}

\author{
Jade Vande Kamp \\ Vibration Research, Jenison, MI, United States \\ E-mail:vvrsales@vibrationresearch.com
}

Received 14 September 2017; accepted 23 September 2017 DOI https://doi.org/10.21595/vp.2017.19184

\begin{abstract}
Today's vibration testing standards are intended to simulate the field environments a product, device or package will experience in the real world. Products are tested for a wide range of reasons, from $\mathrm{R} \& \mathrm{D}$, to production, to life testing. Understanding how a product will react to the real world and the end use environment is critical. It is common practice to collect field data from the end-use environment to verify the testing conditions have accounted for all the real-world conditions. This paper and presentation will discuss the variety of testing options available for the collected data. This includes playing back the recorded data on a shaker in real time, also referred to as long time-history playback. Other options discussed will be FFT transformation with average and peak-hold methods, the influence of kurtosis on the recorded data, and the proper use of the fatigue damage spectrum to generate a random PSD.
\end{abstract}

Keywords: field data, vibration test, accelerometer, field data replication, fatigue damage spectrum.

\section{Collecting field data}

Collecting data is essential for testing. This data can be used to validate existing testing methods, to create new tests, and even be directly replicated on a shaker. Collecting valid data is even more critical. When collecting data, it is important to use the proper accelerometers, mounting techniques and sample rate. When selecting an accelerometer, it is important to use an accelerometer that will not saturate in the environment being recorded. Most IEPE accelerometers have a $\pm 5 \mathrm{~V}$ signal. This means that a $100 \mathrm{mV} / \mathrm{G}$ accelerometer typically saturates at approximately $50 \mathrm{G}$ 's of acceleration and a $10 \mathrm{mV} / \mathrm{G}$ accelerometer at $500 \mathrm{G}$ 's.

Next, when mounting an accelerometer, it is important to use an appropriate method and location. Beeswax is a common mounting method for quick acquisition, but is not the most accurate method. Stud mounting an accelerometer is by far the best method of mounting a transducer. Using an appropriate adhesive/cement to fix the accelerometer is the next best option. Other methods like beeswax and magnetic mounting significantly lower the resonance of the accelerometer due to the poor connection between the accelerometer and the device under test (DUT). This can have significant effects on a recording. Selecting an appropriate mounting position is also important when the goal is laboratory analysis and re-creation. It is best to position the accelerometer in the axis of the predominate vibration as that is the position that it will be tested in the laboratory. When testing in the laboratory be sure to mount the accelerometer in the same location and orientation as originally recorded. It is also important to properly secure the cables to prevent cable whip and connector strain. There is a phenomenon known as triboelectric effect, in which a cable whip can induce noise, especially in high-impedance signal paths. Cable strain near either electrical connector can lead to intermittent or broken connections and a loss of data. There are many other considerations that must be made when recording data. The number of channels, battery life, and sample rate can affect the maximum recording time. An end use environment with high frequency content will also increase the peaks and RMS content of the recording. High frequency data often has little effect on the product in the real world and can be filtered out when post-processing the data and creating a test profile.

\section{Creating a test with real world data}

There are multiple methods that can be used to generate vibration test profiles based on real 
world data. Field Data Replication (long time history playback), Random Import and Fatigue Damage Spectrum are common methods of generating tests. Field Data Replication is the most "real world" test method used. It is an exact 1:1 replication of what was recorded in the field. This test method can be very time consuming. The data is played back $1: 1$, this means that the data is not randomized, and typically is not accelerated. It is possible to increase the levels of the test to increase the amplitude, but there is still no randomization. These types of tests are often used when analyzing the response of a product to a particular environment. The most common application is Buzz, Squeak and Rattle (BSR) testing where the engineer is listening for anything within the human hearing range that could be excited by a field environment.

There are two control methods used across the industry. The first is an iterative algorithm that provides a pseudoclosed loop control. The waveform is played at a low level, then scaled/incremented. This process is repeated until the entire playback falls within the required tolerances based on voltage measurements. Although this process does work, while the waveform is "playing" there is not true, closed-loop, feedback control. There are many cases where a product fails, or an accelerometer falls off and the test continues to run until reaching the end of the waveform. The other method of control is a predictive method. This requires a controller with significant processing power and dynamic range, but results in a much more reactive test when changes or failures occur. This process uses true closed loop, feedback control and will react to failures by shutting down the test. This can prevent significant damage to the DUT, shaker and transducers.

Random import is a software tool that analyzes the field recording and creates a random test profile based on the data. There are two common methods used for this analysis: Average and Peak hold. The peak hold and average options divide the file into blocks, compute the spectrum for each block, and then combine the using the selected method. The average method averages all the spectra together, creating a uniform average. The peak hold method extracts the peak values for each frequency band over all the blocks at each frequency. The average method combines the FFT where peak hold concentrates the energy on the peak acceleration for a section of time. These methods provide a valuable analysis and can be used to generate a random test profile. The methods used are commonly accepted in many industries, but there are some engineering justifications that need to be made especially when an environment is non-Gaussian. These methods used a combination of FFT's to create the random PSD. In a non-Gaussian environment, using the Average Method, the high peaks are averaged out and not properly represented in the calculation, resulting in an under test. The same thought can be applied to the Peak Hold method, but in this case the peaks are over emphasized resulting in an over test. There are several questions that an engineer still must answer when using either of these methods: Is it possible to combine multiple environments into a single test? Is it possible to reliably accelerate these tests? Is it possible to create a test profile that is the damage equivalent to a field environment?

Analyzing an environment or series of environments using the Fatigue Damage Spectrum (FDS) and then converting the resultant FDS's to create a PSD is an accurate method of determining the appropriate test profile for a single environment or multiple environments. Although this method has assumptions that need to be overcome, it is gaining popularity as the method used to generate and reliably accelerate random tests that are based on field environments. The FDS, when generated via a cycle counting and Rainflow analysis method, accounts for any non- Gaussian nature of the field environments, and maintains a time-based relationship between the original field data, the FDS and the resultant PSD. This time base relationship allows for reliable test acceleration. There are other methods that can be used to generate a FDS other than Rainflow analysis. It is possible to generate a FDS from an PSD. This method is applicable IF the data being analyzed is Gaussian in nature. The conversion from PSD to FDS assumes a Gaussian output. If the original field data is not Gaussian in nature, then the resultant FDS will be much different than those generated through the Rainflow process. The non-Gaussian nature of many industries create a much more damaging environment due to the increase in peak acceleration. Properly accounting for the damage created by these peaks is essential to creating a test that is 
representative of the environments being analyzed. If necessary or desirable, it is possible to re-introduce kurtosis into the random test that is generated to ensure that the peak acceleration of the test is equivalent to the peak acceleration from the real world.

There are two main parameters associated with the fatigue damage spectrum. The first is the material property $(\mathrm{m})$. This is the slope of an idealized S-N curve when plotted on a log-log graph. If a precise $\mathrm{m}$ value is not known, estimations can be made. Most metals have a m value between 4 and 8 , to increase the overall damage calculated at a frequency a lower $m$ value can be used. A low $\mathrm{m}$ value (e.g. 2) will result in a higher damage number for each frequency when compared to a higher $m$ value (e.g. 10). If the product passes a test generated using a lower $m$ value, it should survive a test generated at a higher value. The second parameter is the quality factor $(\mathrm{Q})$ of the narrowband filters that separate the bins of the spectrum. It is possible to control how sharp or shallow the transitions in the fatigue damage spectrum are by adjusting the value of $\mathrm{Q}$. It is recommended that the $\mathrm{Q}$ be greater than or equal to the $\mathrm{Q}$ of the primary resonance of the product to ensure that the damage at that resonance is properly accounted for. Target life and test duration are the final elements required to generate a test profile. Each recording has a target life (time or number of repetitions) to scale to the partial contribution a waveform represents in the overall lifetime of the product. The total target life is the sum of each individual waveforms target life. The test duration can be changed to accelerate the test. The time-based method used to generate the FDS allows for reliable test acceleration and proper accounting for any non-Gaussian nature of the time history files. As a test is accelerated, it does add some inaccuracy to the calculation. The ratio between the target life and the test duration should be set appropriately so as not to cause instantaneous shock on the product, and applying high cycle fatigue to the product.

\section{Conclusions}

There are many methods and tests used today that are generated using field recorded data. First and foremost, it is critical to have data that is useful. Ensuring that the data is valid and accurate allows a valid and accurate test to be generated. Using the proper tools and methodology to acquire that data makes this simple and repeatable. Next, when creating a test, it is important to understand the type of test that is best for the requirements. Creating a lifetime of damage in a Field Data Replication test is simply unrealistic if your product has thousands of hours of lifetime expectancy. Using a high amplitude random test to observe the audible response of a product to its real-world environment will not result in any useful conclusions. Finally, using the appropriate calculation and control methodology for any test profile will result in a test that is applicable and allows engineers to draw useful conclusions.

\section{References}

[1] Accelerometer Mounting Considerations. http://www.dytran.com/assets/PDF/Accelerometer\% 20Mounting\%20Considerations.pdf.

[2] Connecting Different Sensors to your VR Controller. http://www.vibrationresearch.com /training_video/Webinar/powerpoints/connectingdifferentsensors_v2014.pdf.

[3] Field to Report Webinar. 2013, http://www.vibrationresearch.com/training_video/Webinar /powerpoints/Field_to_Lab_FDS_v11.pdf. 